\title{
Medical errors and their prevention
}

\author{
Mariusz Tybinski*, Pavlo Lyovkin, Veronika Sniegirova, Daniel Kopec
}

Computer and Information Science, Brooklyn College, New York City, USA; 'Corresponding Author: mariusz.tybinski@gmail.com

Received 24 December 2011; revised 8 January 2012; accepted 20 February 2012

\begin{abstract}
New protocols and procedures for reducing medical errors will be discussed and evaluated in this paper. Further Computerized Physician Order Entry and Clinical Decision Support Systems coupled with monitoring of adverse drug events will be explored. Then lastly some common mistakes of "Wrong-Side" type surgeries and methods to prevent them will be discussed.
\end{abstract}

Keywords: Medical Errors; Mishaps; Complications; CPOE; CDSS; Wrong-Side Surgeries; WSS;

19-Item Checklist Study

\section{INTRODUCTION}

The matter of medical errors is not a new topic and it did not become a widespread subject until the last decade. Statistics show the medical field has improved greatly since IOM's report in 1999; However, those error ratios can still be improved upon or even eliminated completely.

\section{MEDICAL ERRORS AND THEIRPREVENTION}

\subsection{9-Item Checklist Study}

In the study published in 2009 by The New England Journal of Medicine it was reported that implementation of the Surgical Safety Checklist (based on the first edition of the World Health Organization Guidelines for Safe Surgery) helped eliminate some surgical complications and mishaps.

\subsection{The Checklist}

The Back in 2008 the World Health Organization published recommended guidelines, principles, and recommended practice to improve the safety of patients. Based on WHO's guidelines, a 19-Item check list was designed with the intention of reducing the rate of major surgical complications and mishaps [1].

The implementation involved a two step process. First, the initial baseline data was collected, and then each local investigator was advised of the analyzed deficiencies and asked to implement the 19-Item check list (Table 1).

This study was conducted in eight participating hospitals within the WHO region (Table 2). For each hospital a local data collector was chosen and trained accordingly (Table 3). This employee worked full time on the conducted study and was not assigned any clinical or hospital duties at the time. The list was introduced to the staff by lectures, written material, or direct training. Each hospital designated between 1-to-4 operating rooms and all patients of 16 years of age and older ware consecutively enrolled [1].

\subsection{Observations and Data Gathering}

The study data was collected from the individual local collectors and/or from the clinical teams involved. The information gathered from surgery recovery period included demographics of patients, procedural safety, and type of the anesthetic data. The information was collected primarily from patient's first 30 days or until discharge. The collection of data aimed for 500 consecutively enrolled patients [1].

The researchers' defined complications included "acute renal failure, bleeding requiring the transfusion of 4 or more units of red cells within the first 72 hours after surgery, cardiac arrest requiring cardiopulmonary resuscitation, coma of 24 hours' duration or more, deep-vein thrombosis, myocardial infarction, unplanned intubation, ventilator use for 48 hours or more, pneumonia, pulmonary embolism, stroke, major disruption of wound, infection of surgical site, sepsis, septic shock, the systemic inflammatory response syndrome, unplanned return to the operating room, vascular graft failure, and death" [1].

\subsection{9-Item Checklist Results}

In this study a $20 \%$ reduction in complications was found by the researchers after the checklist was implemented with a statistical power of $80 \%$.

There were 3733 enrolled patients during the baseline period and 3955 patients after the introduction of the 19-Item check list (Table 4).

The error complications for any of the sites dropped to $7 \%$ from $11 \%$ (at the initial baseline) on the average of every site, and the total in-hospital rate of death dropped 
Table 1. Elements of the surgical safety checklist ${ }^{*}$.

$>>$ Sign in

1. Before induction of anesthesia, members of the team (at least the nurse and an anesthesia professional) orally confirm that:

- The patient has verified his or her identity, the surgical site and procedure, and consent;

- The surgical site is marked or site marking is not applicable;

- The pulse oximeter is on the patient and functioning;

- All members of the team are aware of whether the patient has a known allergy;

- The patient's airway and risk of aspiration have been evaluated and appropriate equipment and assistance are available;

- If there is a risk of blood loss of at least $500 \mathrm{ml}$ (or $7 \mathrm{ml} / \mathrm{kg}$ of body weight, in children), appropriate access and fluids are available. $>$ Time out

2. Before skin incision, the entire team (nurses, surgeons, anesthesia professionals, and any others participating in the care:

o of the patient) orally;

o Confirms that all team members have been introduced by name and role;

o Confirms the patient's identity, surgical site, and procedure;

o Reviews the anticipated critical events;

o Surgeon reviews critical and unexpected steps, operative duration, and anticipated blood loss;

o Anesthesia staff review concerns specific to the patient;

o Nursing staff review confirmation of sterility, equipment availability, and other concerns;

o Confirms that prophylactic antibiotics have been administered $\leq 60 \mathrm{~min}$ before incision is made or that antibiotics are not indicated;

o Confirms that all essential imaging results for the correct patient are displayed in the operating room. $>$ Sign out

3. Before the patient leaves the operating room:

o Nurse reviews items aloud with the team;

o Name of the procedure as recorded;

o That the needle, sponge, and instrument counts are complete (or not applicable);

o That the specimen (if any) is correctly labeled, including with the patient's name;

o Whether there are any issues with equipment to be addressed;

The surgeon, nurse, and anesthesia professional review aloud the key concerns for the recovery and care of the patient.

* The checklist is based on the first edition of the WHO Guidelines for Safe Surgery (also available with the full text of this article at NEJM.org).

Table 2. Characteristics of participating hospitals.

\begin{tabular}{|c|c|c|c|c|}
\hline Site & Location & No. of Beds & $\begin{array}{c}\text { No. of } \\
\text { Operating Rooms }\end{array}$ & Type \\
\hline Prince Hamzah Hospital & Amman, Jordan & 500 & 13 & Public, urban \\
\hline St. Stephen's Hospital & New Delhi, India & 733 & 15 & Charity, urban \\
\hline University of Washington Medical Center & Seattle, Washington & 410 & 24 & Public, urban \\
\hline St. Francis Designated District Hospital & Ifakara, Tanzania & 371 & 3 & District, rural \\
\hline Toronto General Hospital & Toronto, Canada & 744 & 19 & Public, urban \\
\hline St. Mary’s Hospital ${ }^{*}$ & London, England & 541 & 16 & Public, urban \\
\hline Auckland City Hospital & Auckland, New Zealand & 710 & 31 & Public, urban \\
\hline
\end{tabular}

${ }^{*}$ St. Mary's Hospital has since been renamed St. Mary's Hospital_-Imperial College National Health Service Trust [1].

Table 3. Surgical safety policies in place at participating hospitals before the study.

\begin{tabular}{|c|c|c|c|c|c|c|}
\hline \multirow[b]{2}{*}{ Site No. } & \multirow{2}{*}{$\begin{array}{c}\text { Routine } \\
\text { Intraoperative } \\
\text { Monitoring with } \\
\text { Pulse Oximetry }\end{array}$} & \multirow{2}{*}{$\begin{array}{l}\text { Oral Confirmation of } \\
\text { Patient's Identity and } \\
\text { Surgical Site in } \\
\text { Operating Room }\end{array}$} & \multirow{2}{*}{$\begin{array}{c}\text { Routine Administration } \\
\text { of Prophylactic Antibiotics } \\
\text { in Operating Room }\end{array}$} & \multirow{2}{*}{$\begin{array}{l}\text { Standard Plan for } \\
\text { Intravenous Access } \\
\text { for Cases of High } \\
\text { Blood Loss }\end{array}$} & \multicolumn{2}{|c|}{ Formal Team Briefing } \\
\hline & & & & & Preoperative & Postoperative \\
\hline 1 & Yes & Yes & Yes & No & No & No \\
\hline 2 & Yes & No & Yes & No & No & No \\
\hline 3 & Yes & No & Yes & No & No & No \\
\hline 4 & Yes & Yes & Yes & No & No & No \\
\hline 5 & No & No & No & No & No & No \\
\hline 6 & No & No & Yes & No & No & No \\
\hline 7 & Yes & No & No & No & No & No \\
\hline 8 & Yes & No & No & No & No & No \\
\hline
\end{tabular}

* Sites 1 through 4 are located in high-income countries; Sites 5 through 8 are located in low- or middle-income countries [1]. 
Table 4. Characteristics of the patients and procedures before and after checklist implementation, according to site* .

\begin{tabular}{ccccccccccccc}
\hline Site No. & $\begin{array}{c}\text { No. of Patients } \\
\text { Enrolled }\end{array}$ & \multicolumn{2}{c}{ Age } & \multicolumn{2}{c}{ Female Sex } & \multicolumn{2}{c}{ Urgent Case } & \multicolumn{2}{c}{$\begin{array}{c}\text { Outpatient } \\
\text { Procedure }\end{array}$} & \multicolumn{2}{c}{ General Anesthetic } \\
\hline Column1 & Before & After & Before 2 & After 3 & Before 4 & After 5 & Before 6 & After 7 & Before 8 & After 9 & Before 10 & After 11 \\
\hline 1 & 524 & 598 & $51.9 \pm 15.3$ & $51.4 \pm 14.7$ & 58.2 & 62.7 & 7.4 & 8 & 31.7 & 31.8 & 95 & 95.2 \\
2 & 357 & 351 & $53.5 \pm 18.4$ & $54.0 \pm 18.3$ & 54.1 & 56.7 & 18.8 & 14.5 & 23.5 & 20.5 & 92.7 & 93.5 \\
3 & 497 & 486 & $51.9 \pm 21.5$ & $53.0 \pm 20.3$ & 44.3 & 49.8 & 17.9 & 22.4 & 6.4 & 9.3 & 91.2 & 94 \\
4 & 520 & 545 & $57.0 \pm 14.9$ & $56.1 \pm 15.0$ & 48.1 & 49.6 & 6.9 & 1.8 & 14.4 & 11 & 96.9 & 97.8 \\
5 & 370 & 330 & $34.3 \pm 15.0$ & $31.5 \pm 14.2$ & 78.3 & 78.4 & 46.1 & 65.4 & 0 & 0 & 17 & 10 \\
6 & 496 & 476 & $44.6 \pm 15.9$ & $46.0 \pm 15.5$ & 45 & 46.6 & 28.4 & 22.5 & 1.4 & 1.1 & 61.7 & 59.9 \\
7 & 525 & 585 & $37.4 \pm 14.0$ & $39.6 \pm 14.9$ & 69.1 & 68.6 & 45.7 & 41 & 0 & 0 & 49.1 & 55.9 \\
8 & 444 & 584 & $41.9 \pm 15.8$ & $39.7 \pm 16.2$ & 57 & 52.7 & 13.5 & 21.9 & 0.9 & 0.2 & 97.5 & 94.7 \\
Total & 3733 & 3955 & $46.8 \pm 18.1$ & $46.7 \pm 17.9$ & 56.2 & 57.6 & 22.3 & 23.3 & 9.9 & 9.4 & 77 & 77.3 \\
P value & & & & & 0.63 & 0.21 & & 0.26 & & 0.4 & & 0.68 \\
\hline
\end{tabular}

*Plus-minus values are means \pm SD. Urgent cases were those in which surgery within 24 hours was deemed necessary by the clinical team. Outpatient procedures were those for which discharge from the hospital occurred on the same day as the operation. P values are shown for the comparison of the total value after checklist implementation with the total value before implementation [1].

to. $08 \%$ from $1.5 \%$ (Table 5). The surgical complications and death rate fell on average by $36 \%$. Every hospital had a decrease of major postoperative complications with a significant reduction at three sites (one in a high-income location and two in lower-income locations). Even though in some hospitals a few effects of the intervention were stronger than the others, no single hospital was responsible for the overall rate change, nor was the rate limited exclusively to a high or low income hospital. From these findings it was concluded that the checklist program is an improvement among diverse clinical and economical environments.

\section{COMPUTERIZED PHYSICIAN ORDER ENTRY (CPOE) AND CLINICALDECISION SUPPORT SYSTEMS (CDSS) OVERVIEW}

One of the most common, clinically important and costly problems is Medication errors and adverse drug events (ADEs) - injuries that result from the use of a drug [2]. Both adult and pediatric studies have found that about half of all medication errors occur during the drug ordering, although another technique-direct observation, has shown that errors also occur during the administration stage. The principle types of medication errors are: missing a dose, incorrect dose, route or frequency of taking the medications. The introduction of computerized physician order entry (CPOE) with clinical decision support systems (CDSSs) may reduce medication-related errors [3].

Complete and standard formatted orders are only accepted by the basic CPOE systems. Almost all modern CPOE systems are somehow integrated with CDSSseither include complete CDSSs or have an interface. Clinical decisions can include values or suggestions for drug routes, frequencies, doses. More advanced CDSSs perform allergy, interaction and value checks. They can also provide reminders about orders and other useful features.

The second type of CDSSs is called isolated CDSSs. They are stand-alone systems that provide advice on selection, dosage, and duration or drugs. More refined CDSSs are using patient-specific information and their advice is based on this information. After using CDSS system, the physician has to proceed with a handwritten medication order.

It is estimated that about 780,000 patients were injured or died in hospitals across the US from ADEs annually. Based on studies by a few US hospitals, incidence rates of ADEs range between 3 to 7 per every 100 admissions. Researchers use different definitions and criteria and that is why it is difficult to calculate a national estimate. One study demonstrated that $57 \%$ OCEs occurred at the ordering stage, 34\%-administration stage, $7 \%$-transcribing stage, and 3\%-dispensing [3].

\subsection{CPOE and CDSS Study Designs}

This paper reviews four studies that evaluate CPOE with CDSSs. The first study is a randomized controlled trial that evaluates utility of CPOE in improving prescriptions. This study was done by the Regenstrief Institute for Health Care. Other three studies evaluate CPOE systems at Brigham and Women's Hospital (BWH). The first BWH study compares a period of intervention of CPOE with CDSSs on surgical, intensive and medical care wards with a historical period. The other two studies are time-based analysis of orders [4].

Table 6(b) shows four studies that evaluate isolated CDSSs.

The first BWH study demonstrated a $55 \%$ decrease in non-intercepted serious medication errors. This study 
Table 5. Outcomes before and after checklist implementation, according to site*.

\begin{tabular}{|c|c|c|c|c|c|c|c|c|c|c|c|c|}
\hline \multirow[b]{2}{*}{ Site No. } & \multicolumn{2}{|c|}{$\begin{array}{l}\text { No. of Patients } \\
\text { Enrolled }\end{array}$} & \multicolumn{2}{|c|}{$\begin{array}{l}\text { Surgical-Site } \\
\text { Infection }\end{array}$} & \multicolumn{2}{|c|}{$\begin{array}{c}\text { Unplanned Return } \\
\text { to } \\
\text { the Operating Room }\end{array}$} & \multicolumn{2}{|c|}{ Pneumonia } & \multicolumn{2}{|c|}{ Death } & \multicolumn{2}{|c|}{ Any Complication } \\
\hline & Before & After & Before 2 & After 3 & Before 4 & After 5 & Before 6 & After 7 & Before 8 & After 9 & Before 10 & After 11 \\
\hline 1 & 524 & 598 & 4 & 2 & 4.6 & 1.8 & 0.8 & 1.2 & 1 & 0 & 11.6 & 7 \\
\hline 2 & 357 & 351 & 2 & 1.7 & 0.6 & 1.1 & 3.6 & 3.7 & 1.1 & 0.3 & 7.8 & 6.3 \\
\hline 3 & 497 & 486 & 5.8 & 4.3 & 4.6 & 2.7 & 1.6 & 1.7 & 0.8 & 1.4 & 13.5 & 9.7 \\
\hline 4 & 520 & 545 & 3.1 & 2.6 & 2.5 & 2.2 & 0.6 & 0.9 & 1 & 0.6 & 7.5 & 5.5 \\
\hline 5 & 370 & 330 & 20.5 & 3.6 & 1.4 & 1.8 & 0.3 & 0 & 1.4 & 0 & 21.4 & 5.5 \\
\hline 6 & 496 & 476 & 4 & 4 & 3 & 3.2 & 2 & 1.9 & 3.6 & 1.7 & 10.1 & 9.7 \\
\hline 7 & 525 & 585 & 9.5 & 5.8 & 1.3 & 0.2 & 1 & 1.7 & 2.1 & 1.7 & 12.4 & 8 \\
\hline 8 & 444 & 584 & 4.1 & 2.4 & 0.5 & 1.2 & 0 & 0 & 1.4 & 0.3 & 6.1 & 3.6 \\
\hline Total & 3733 & 3955 & 6.2 & 3.4 & 2.4 & 1.8 & 1.1 & 1.3 & 1.5 & 0.8 & 11 & 7 \\
\hline$P$ value & & & \multicolumn{2}{|c|}{$<0.001$} & \multicolumn{2}{|c|}{0.047} & \multicolumn{2}{|c|}{0.46} & \multicolumn{2}{|c|}{0.003} & \multicolumn{2}{|c|}{$<0.001$} \\
\hline
\end{tabular}

*The most common complications occurring during the first 30 days of hospitalization after the operation are listed. Bold type indicates values that were significantly different (at $\mathrm{P}<0.05$ ) before and after checklist implementation, on the basis of $\mathrm{P}$ values calculated by means of the chisquare test or Fisher's exact test. P values are shown for the comparison of the total value after checklist implementation as compared with the total value before implementation [1].

Table 6(a). Studies of computerized physician order entry (CPOE) with clinical decision support systems (CDSSs) ${ }^{*}$.

\begin{tabular}{|c|c|c|c|}
\hline Study & Study Design & Study Outcomes & Results \\
\hline $\begin{array}{l}\text { Overhage, 1997. Impact of faculty and physician } \\
\text { reminders (using CPOE) on corollary orders for } \\
\text { adult inpatients in a general medical ward at a public } \\
\text { teaching hospital affiliated with the Indiana } \\
\text { University School of Medicine }\end{array}$ & $\begin{array}{l}\text { Level } 1 \text { (RCT with } \\
\text { physicians randomized } \\
\text { to receive reminders or } \\
\text { not) }\end{array}$ & $\begin{array}{l}\text { Levels } 2 \& 3 \text { (errors of } \\
\text { omission in corollary orders) }\end{array}$ & $\begin{array}{l}25 \% \text { improvement in ordering of } \\
\text { corollary medications by faculty and } \\
\text { residents }(\mathrm{P}<0.0001)\end{array}$ \\
\hline $\begin{array}{l}\text { Bates, 1998. CPOE with CDSSs for adult } \\
\text { inpatients on medical, surgical, and intensive care } \\
\text { wards at BWH, a tertiary care center affiliated with } \\
\text { Harvard University }\end{array}$ & $\begin{array}{l}\text { Levels } 2 \& 3 \text { (two } \\
\text { study designs) }\end{array}$ & $\begin{array}{l}\text { Level } 1 \text { (ADE rates) and Level } 2 \\
\text { (serious medication errors) }\end{array}$ & $\begin{array}{l}55 \% \text { decrease in non-intercepted } \\
\text { serious medication errors }(\mathrm{P}=0.01) \\
17 \% \text { decrease in preventable ADEs } \\
(\mathrm{P}=0.37)\end{array}$ \\
\hline $\begin{array}{l}\text { Bates, 1999. CPOE with CDSSs for adult } \\
\text { inpatients in } 3 \text { medical units at BWH }\end{array}$ & $\begin{array}{l}\text { Level } 3 \text { (retrospective } \\
\text { time series) }\end{array}$ & $\begin{array}{l}\text { Level } 1 \text { (ADEs) and Level } 2 \\
\text { (main outcome measure was } \\
\text { medication errors) }\end{array}$ & $\begin{array}{l}81 \% \text { decrease in medication errors }(\mathrm{P} \\
<0.0001) \\
86 \% \text { decrease in non-intercepted } \\
\text { serious medication errors }(\mathrm{P}=0.0003)\end{array}$ \\
\hline $\begin{array}{l}\text { Teich, 2000. CPOE with CDSSs for all } \\
\text { adult inpatients at BWH }\end{array}$ & $\begin{array}{l}\text { Level } 3 \text { (retrospective } \\
\text { before-after analysis) }\end{array}$ & $\begin{array}{l}\text { Levels } 2 \& 3 \text { (changes in } 5 \\
\text { prescribing practices) }\end{array}$ & $\begin{array}{l}\text { Improvement in } 5 \text { prescribing } \\
\text { practices }(\mathrm{P}<0.001 \text { for each of the } 5 \\
\text { comparisons })\end{array}$ \\
\hline
\end{tabular}

*ADE indicates adverse drug event; BWH—Brigham and Women's Hospital; and RCT—randomized controlled trial.

Table 6(b). Studies of clinical decision support systems (CDSSs) ${ }^{*}$.

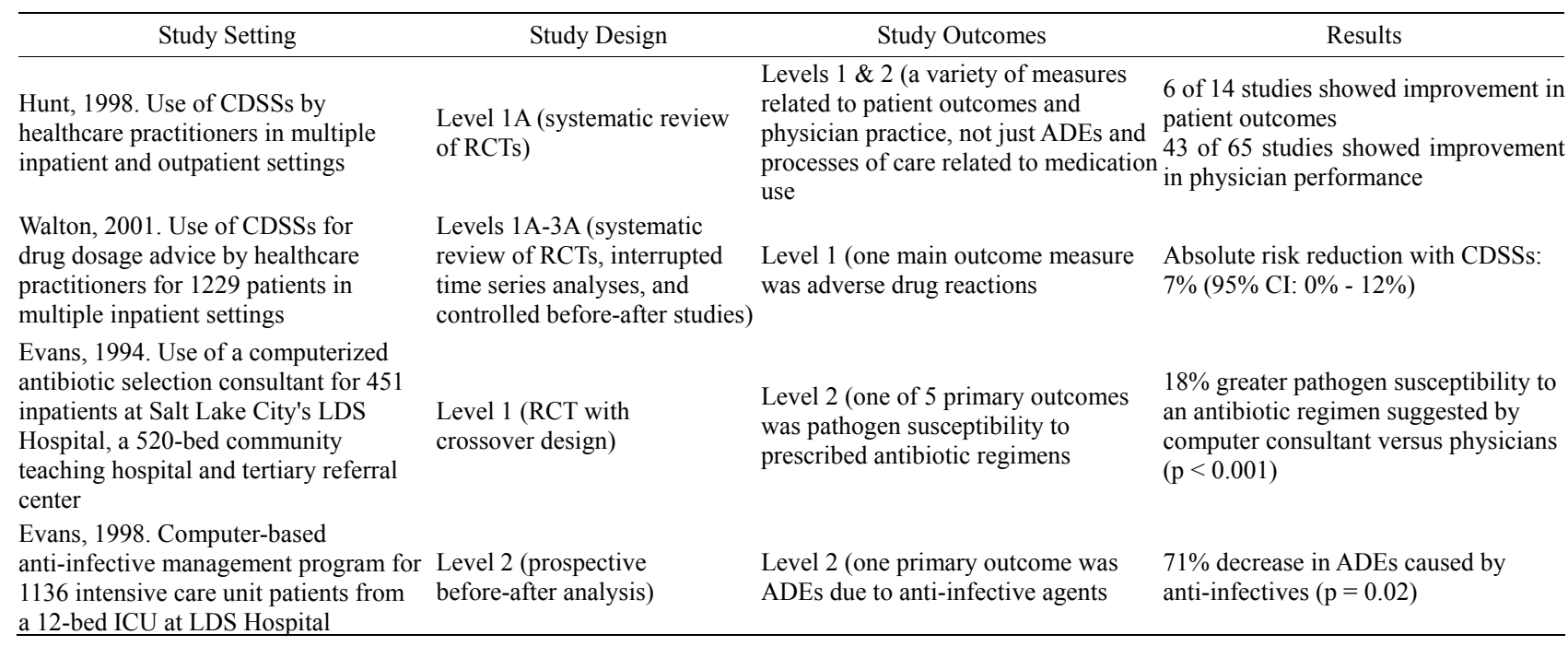
a 12-bed ICU at LDS Hospital

*ADE indicates adverse drug event; CI — confidence interval; ICU—intensive care unit; and RCT—randomized controlled trial. 
also found a $17 \%$ decrease in preventable ADEs. The second study, showed marked reductions in all errors except dose errors and in non-intercepted serious errors. The number of ADEs was small-only 25 during the baseline. The total number of ADEs per thousand patient days decreased from 14.5 to $9.5(\mathrm{p}=0.09)$. For preventable ADEs, the reduction was 0.05 [5].

Both Overhage and Teich studies demonstrate the evidence that CPOE with CDSSs can decrease medication errors in all areas. At the same time the reduction in ADEs did not achieve any significance in one study, and only achieved some in one of the outcomes in the other study [6]. Furthermore, the all the systems were developed internally and were not purchased.

In the studies evaluating CDSSs, two were systematic reviews. Hunt's review showed that 6 of 14 studies demonstrated improvement in patient outcomes and 43 of 65 studies demonstrated improvement in physician performance. The conclusion here is that CDSSs can enhance preventive care, drug dosage, other aspects of medical care performance, but the CDSS's impact on patient outcomes is still unclear. Walton's review evaluated computerized drug dosage and showed a 7\% decrease in adverse drug reactions. The 1994 Evans study evaluated the use of a computerized antibiotic selection consultant, and showed an $18 \%$ greater susceptibility to an antibiotic suggested by an electronic system versus a physician. The 1998 study by Evans demonstrated a 71\% decrease in ADEs caused by anti-infectives when using a computer based anti-infective systems [7]. These CDSSs studies showed improvements in medical errors with significance.

\subsection{Potential for Harm}

While CPOE and CDSS systems proved themselves to be very effective systems for reducing medical errors, they may also cause additional problems. For example, an incorrect dosage suggestion may lead to an incorrect ordering choice by the physician.

There were Also cases where a physician would accidently write an order in the wrong patient records.

In addition, alarm systems should be appropriately balanced between sensitivity and specificity. Physicians should receive a warning whenever a potential harm is possible, but at the same he/she should not be overwhelmed by many "false positive" alerts. Hardware and software outage should also be considered.

\subsection{Costs and Benefits}

Six of the studies evaluated in this paper are known as "home-grown" systems. The costs of commercial systems are significantly greater than the costs of developing the same systems. For BWH, the CPOE development and implementation cost was estimated to be $\$ 1.9$ million, with a maintenance cost of $\$ 500,000$ annually. At the same time, the cost of complete commercial systems varies and may be on the order of tens of millions of dollars [8].

While everyone recognizes that $\mathrm{CPOE}$ systems require a significant capital investment, research has shown that the average return on investment for such systems can range from $\$ 180,000$ - $\$ 900,000$ annually [8]. Furthermore, with some additional improvements in reduction in testing and imaging as well as resource utilization, some hospitals can save millions of dollars annually.

\section{WRONG-SITE SURGERIES}

A wrong-side surgery is a surgery that was made on the wrong site of the body or for a wrong patient. This problem became even more prevalent for the past decade. During the Joint Commission's Sentinel Event program the number of wrong-side surgeries (WSS) that were reported increased from 15 cases in 1998 to a total number of 592 cases by June 30, 2007 [9]. "Incidence, patterns, and prevention of wrong-site surgery" study states that these kind of errors happen once in 112,994 cases [10]. For instance the New York State Department of Health reported 46 cases in 2 years using a mandatory reporting system. A survey of hand surgeons stated that $20 \%$ of the 100 respondents had operated on the wrong site at least once during their professional career and even $16 \%$ of them had prepared to operate on the wrong site, but realized their error before making an incision [11]. However this number of errors is much higher out of operating rooms, for example they may occur during ambulatory surgery or interventional radiology.

Wrong-site surgeries usually occur in orthopedic or podiatric procedures, general procedures, urological and neurosurgical procedures.

So why do we pay so much attention to them if wrongsite surgeries are approximately rare $(1: 100,000)$ and most of them $(64 \%)$ do not result in permanent injury?

WSS are pretty important as they are a result of some previous small failures and become a symptom of the systems and culture of the health care. Where by fixing them we will address a much bigger problem for health care.

Failures that result in a WSS may occur any moment during the time a patient has entered a hospital until a surgery has completed. Poor handwriting, wrong medical record review, wrong patient assessment, lack of communication among operative team are key problems. A chance of error may increase when multiple procedures are operated on the same patient, when the operative team is under time pressure and some previous changes were made to the operative team. 


\subsection{Ways of Preventing a WSS}

Initial attempts to avoid WSS were focused on developing an awareness mechanism for identifying a correct site. American Academy of Orthopedic Surgeons developed a campaign to prevent WSS by marking the correct surgical site that was named "Sign Your Site" [12]. Apparently a simple process was actually confusing doctors because they often thought that the marked site was the site where they should not operate. So later on to make this program more effective doctors had to mark an incorrect site with word "No" and after that a surgical team had to work together to verify the correct site.

A summit of Joint Commission in 2003 brought many health care professionals and others to discover a way to reduce or eliminate WSS. This summit was devoted to problems of WSS impact on patients, their families and health care professionals and had to address continued escalation of reported WSS cases. As a result of this summit The Universal Protocol for Preventing Wrong Site, Wrong Procedures, and Wrong Person Surgery TM was created [13].

\subsection{The Universal Protocol}

The Universal Protocol requires a critical 3-step process: "A pre-procedure verification, marking the correct surgical site and a "time-out" for the operating stuff just before the surgery". For the study Stanhel's team was able to collect surgical errors data from the company that provides liability insurance to 6000 doctors in Colorado [13].

The Universal Protocol has to be used in ambulatory care, hospitals, critical access hospitals, and office-based settings. It was first used on July 1, 2004 when all three key elements were included in accreditation process for health care organizations.

The Universal Protocol is stated below in Text Box 1.

The Joint Commission Universal Protocol for Preventing Wrong Site, Wrong Procedure, Wrong Person Surgery TM.

\subsection{Reports}

The collected data by the authors indicates 27,370 reported events between January 2002 and June 2008 and among them the researchers found 25 wrong-patient and 107 wrong-side operations. From 25 reported patients, 5 received unnecessary surgeries and 38 patients (from 107 wrong-side reported patients) received operations that significantly harmed patients' health - including one death.

There was an $85 \%$ judgment error rate for the operations on the wrong-side of the body and $72 \%$ due to not performing the "time-out" which was required by the protocol.

\section{Text Box 1.}

Wrong site, wrong procedure, wrong person surgery can be prevented. This Universal Protocol is intended to achieve that goal. It is based on the consensus of experts from the relevant clinical specialties and professional disciplines and is endorsed by more than 40 professional medical associations and organizations.

In developing this protocol, consensus was reached on the following principles:

- Wrong site, wrong procedure, wrong person surgery can and must be prevented.

- A robust approach — using multiple, complementary strategies - is necessary to achieve the goal of eliminating wrong site, wrong procedure, wrong person surgery.

- Active involvement and effective communication among all members of the surgical team is important for success.

- To the extent possible, the patient (or legally designated representative) should be involved in the process.

- Consistent implementation of a standardized approach using a universal, consensus-based protocol will be most effective.

- The protocol should be flexible enough to allow for implementation with appropriate adaptation when required to meet specific patient needs.

- A requirement for site marking should focus on cases involving right/left distinction, multiple structures (fingers, toes), or levels (spine).

- The Universal Protocol should be applicable or adaptable to all operative and other invasive procedures that expose patients to harm, including procedures done in settings other than the operating room.

In concert with these principles, the following steps, taken together, comprise the Universal Protocol for eliminating wrong site, wrong procedure, wrong person surgery:

\section{- Preoperative verification process}

- Purpose: To ensure that all of the relevant documents and studies are available prior to the start of the procedure and that they have been reviewed and are consistent with each other and with the patient's expectations and with the team's understanding of the intended patient, procedure, site, and, as applicable, any implants. Missing information or discrepancies must be addressed before starting the procedure.

Process: An ongoing process of information gathering and verification, beginning with the determination to do the procedure, continuing through all settings and interventions involved in the preoperative preparation of the patient, up to and including the "time out" just before the start of the procedure.

\section{- Marking the operative site}

- Purpose: To identify unambiguously the intended site of incision or insertion.

- Process: For procedures involving right/left distinction, multiple structures (such as fingers and toes), or multiple levels (as in spinal procedures), the intended site must be marked such that the mark will be visible after the patient has been prepped and draped.

- “Time out" immediately before starting the procedure

- Purpose: To conduct a final verification of the correct patient, procedure, site and, as applicable, implants.

- Process: Active communication among all members of the surgical/procedure team, consistently initiated by a designated member of the team, conducted in a "fail-safe" mode, i.e., the procedure is not started until any questions or concerns are resolved.

These guidelines provide detailed implementation requirements, exemptions, and adaptations for special situations.

Preoperative verification process

- Verification of the correct person, procedure, and site should occur (as applicable):

- At the time the surgery/procedure is scheduled.

- At the time of admission or entry into the facility.

- Anytime the responsibility for care of the patient is transferred to another caregiver. 


\section{Continued}

- With the patient involved, awake, and aware, if possible.

- Before the patient leaves the preoperative area or enters the procedure/surgical room.

- A preoperative verification checklist may be helpful to ensure availability and review of the following, prior to the start of the procedure:

- $\quad$ Relevant documentation (e.g., history and physical, consent).

- Relevant images, properly labeled and displayed. o Any required implants and special equipment.

Marking the operative site

- Make the mark at or near the incision site. Do NOT mark any nonoperative site(s) unless necessary for some other aspect of care.

- The mark must be unambiguous (e.g., use initials or "YES" or a line representing the proposed incision; consider that " $\mathrm{X}$ " may be ambiguous).

- The mark must be positioned to be visible after the patient is prepped and draped.

- The mark must be made using a marker that is sufficiently permanent to remain visible after completion of the skin prep. Adhesive site markers should not be used as the sole means of marking the site.

- The method of marking and type of mark should be consistent throughout the organization.

- At a minimum, mark all cases involving laterality, multiple structures (fingers, toes, lesions), or multiple levels (spine). Note: In addition to preoperative skin marking of the general spinal region, special intraoperative radiographic techniques are used for marking the exact vertebral level.

- The person performing the procedure should do the site marking.

- Marking must take place with the patient involved, awake, and aware, if possible.

- Final verification of the site mark must take place during the "time out".

- A defined procedure must be in place for patients who refuse site marking.

Exemptions

- $\quad$ Single organ cases (e.g., Cesarean section, cardiac surgery).

- Interventional cases for which the catheter/instrument insertion site is not predetermined (e.g., cardiac catheterization).

- Teeth-but, indicate operative tooth name(s) on documentation or mark the operative tooth (teeth) on the dental radiographs or dental diagram.

- Premature infants, for whom the mark may cause a permanent tattoo.

"Time out" immediately before starting the procedure Must be conducted in the location where the procedure will be done, just before starting the procedure. It must involve the entire operative team, use active communication, be briefly documented, such as in a checklist (the organization should determine the type and amount of documentation), and must, at the least, include:

- Correct patient identity.

- Correct side and site.

- Agreement on the procedure to be done.

- Correct patient position.

- Availability of correct implants and any special equipment or spcial requirements.

The organization should have processes and systems in place for reconciling differences in staff responses during the "time out."

Procedures for non-OR settings, including bedside procedures

- Site marking must be done for any procedure that involves laterality, multiple structures, or levels (even if the procedure takes place outside of an OR).

- Verification, site marking, and "time out" procedures should be as consistent as possible throughout the organization, including the OR and other locations where invasive procedures are done.

\section{Continued}

Exception: Cases in which the individual doing the procedure is in continuous attendance with the patient from the time of decision to do the procedure and consent from the patient through to the conduct of the procedure may be exempted from the site marking requirement. The requirement for a "time out" final verification still applies.

"Time-out" is an occurrence just before the operation begins where all parties involved check for the right patient and what he or she will be operated on. Stahel notes that the system alone cannot solve any of the surgical related problems in the OR.

The researchers further point out that "Once we were fully responsible for our actions_now we hide behind a safety system that should cover the problem. The time-out is performed, but people are not mentally involved-the system alone cannot protect you from wrong-site surgery". Then he points out that the doctors should take more personal responsibility for their errors stating "We are going from a culture of blame to a culture of system safety, and we should move on to a culture of patient safety and accountability" [13].

An Associate Professor of Surgery at Johns Hopkins University and author of an accompanying journal editorial, Dr. Martin A. Makary said, that "a simple checklist does not solve the problem; it's not that simple. The culture has to change to promote people speaking up when they see a safety concern and promote good teamwork".

Consequently Makary states that often the staff (doctors, nurses etc.) don't even know each other's names and that the "mechanical" culture places doctors above the nurses and other staff. He writes "A nurse or a lowlevel person in the surgical hierarchy may sense that something is not right, but they don't speak up because they are intimidated by the operating room hierarchy." Then he concludes that this culture needs to change and by removing intimidation.

\section{CONCLUSIONS}

Based on the research above we conclude that the number of medical errors has become an increasingly larger concern in the last few decades. Methods like safe practice and universal procedures, checklists, and various computerized systems have proven effective in reduction of medical errors. Even though none of the implemented systems, protocols, or formed checklists helped eliminate those mishaps completely - the data however, clearly shows that error rates have been reduced significantly. Yet there is still a room for improvement, considering people's lives can be saved and hospital financial burden reduced in the near future.

\section{REFERENCES}

[1] Haynes, A.B., Weiser, T.G., Berry, W.R., Lipsitz, S.R., 
Breizat, A.-H.S., Dellinger, P., et al. (2009) A surgical safety checklist to reduce morbidity and mortality in a global population. New England Journal of Medicine, 360, 491-499. doi:10.1056/NEJMsa0810119

[2] Dyer, C.C., Oles, K.S. and Davis, S.W. (1984) The role of the pharmacist in a geriatric nursing home: A literature review. Drug Intelligence \& Clinical Pharmacy, 18, 428-433.

[3] Jenkins, M.H. and Bond, C.A. (1996) The impact of clinical pharmacists on psychiatric patients. Pharmacotherapy, 16, 708-714.

[4] Bates, D.W., Cullen, D.J., Laird, N., Petersen, L.A., Small, S.D., Servi, D., et al. (1995) Incidence of adverse drug events and potential adverse drug events: Implications for prevention. JAMA, 274, 29-34. doi:10.1001/jama.1995.03530010043033

[5] Jha, A.K., Kuperman, G.J., Teich, J.M., Leape, L., Shea, B., Rittenberg, E., et al. (1998) Identifying adverse drug events: Development of a computer-based monitor and comparison with chart review and stimulated voluntary report. Journal of the American Medical Informatics Association, 5, 305-314. doi:10.1136/jamia.1998.0050305

[6] Classen, D.C., Pestotnik, S.L., Evans, R.S., Lloyd, J.F. and Burke, J.P. (1997) Adverse drug events in hospitalized patients. Excess length of stay, extra costs, and at- tributable mortality. JAMA, 277, 301-306. doi:10.1001/jama.1997.03540280039031

[7] Sokol, A.J. (2001) Financial benefits of electronic medical records. www.ehealthcoach.com/FR-FinBenElecMedRec.asp

[8] Joint Commision (2007) Sentinel event statistics as of June 30. http://www.jointcommission.org/sentinel_event.aspx

[9] Kwaan, M.R., Studdert, D.M., Zinner, M.J. and Gawande, A.A. (2006) Archives of Surgery, 141, 353-357.

[10] Meinberg, E.G. and Stern, P.J. (2003) Incidence of wrongsite surgery among hand surgeons. Journal of Bone \& Joint Surgery, 85, 193-197.

[11] American Academy of Orthopedic Surgeons (2006) Wrong site surgery. http://www5.aaos.org/wrong/viewscrp.cfm.

[12] Joint Commission (2003) Universal protocol for preventing wrong site, wrong procedure, wrong person surgery. http://www.jointcommission.org/

[13] Steven, R. (2011) Surgery on wrong patients, surgical sites persists, study finds. http://www.businessweek.com/lifestyle/content/healthday /644585.html 